\title{
Investigation of Metamaterial Coated Conducting Cylinders for Achieving Transparency and Maximizing Radar Cross Section
}

\author{
Erdinc Irci and Vakur B. Ertürk* \\ Dept. of Electrical and Electronics Engineering, Bilkent University, \\ TR-06800 Bilkent, Ankara, Turkey. E-mail: vakur@ee.bilkent.edu.tr
}

\section{Introduction}

Recently, reducing the radar cross sections (RCS) of various structures to achieve transparency and obtaining resonant structures aimed at increasing the electromagnetic intensities, stored or radiated power levels have been investigated [1-4]. The transparency and resonance (RCS maximization) conditions investigated in [1-4] are mainly attributed to pairing of "conjugate" materials: materials which have opposite signs of constitutive parameters [e.g., double-positive (DPS) and doublenegative (DNG) or epsilon-negative (ENG) and mu-negative (MNG)]. In the present work, we extend the transparency and resonance conditions for cylindrical structures when the core cylinder is particularly perfect electric conductor (PEC). The appropriate constitutive parameters of such metamaterials are investigated for both $T E$ and $T M$ polarizations. For $T E$ polarization it is found out that, the metamaterial coating permittivity has to be in the $0<\varepsilon_{c}<\varepsilon_{0}$ interval to achieve transparency, and in the $-\varepsilon_{0}<\varepsilon_{c}<0$ interval to achieve RCS maximization. As in the case of "conjugate" pairing, transparency and resonance are found to be heavily dependent on the ratio of core-coating radii, instead of the total size of the cylindrical structure. However, unlike the "conjugate" pairing cases, replacing $\varepsilon$ by $\mu$ (and vice versa) does not lead to the same conclusions for TM polarization unless the PEC cylinder is replaced by a perfect magnetic conductor (PMC) cylinder. Yet, RCS maximization can also be achieved in the $T M$ polarization case when coating permeability $\mu_{c}<0$, whereas transparency requires large $\left|\mu_{c}\right|$ for this polarization. Numerical results, which demonstrate the transparency and RCS maximization phenomena, are given in the form of normalized monostatic and bistatic echo widths.

\section{Theoretical Background, Transparency and Resonance Conditions}

Consider a PEC cylinder of infinite length, having radius $a$, which is covered by a concentric metamaterial coating with outer radius $b>a$. The metamaterial coating is assumed to be homogeneous and isotropic, having permittivity $\varepsilon_{c}$ and permeability $\mu_{c}$, and it is surrounded by free space $\left(\varepsilon_{0}, \mu_{0}\right)$. The metamaterial coated PEC cylinder is illuminated normally by a uniform plane wave which travels in the direction that makes an angle $\phi_{0}$ with the $+x$ axis. The geometry of the problem is depicted in Fig. 1. For the $T M^{z}$ polarized uniform plane wave, referring to Fig. 1, the incident electric field can be written as $E_{0} e^{-j k_{0} \rho \cos \left(\phi-\phi_{0}\right)}$. Utilizing a similar procedure as in [5], the incident, transmitted and scattered electric fields can be represented respectively as

$$
E_{z}^{i}=E_{0} \sum_{n=-\infty}^{+\infty} j^{-n} J_{n}\left(k_{0} \rho\right) e^{j n\left(\phi-\phi_{0}\right)}
$$




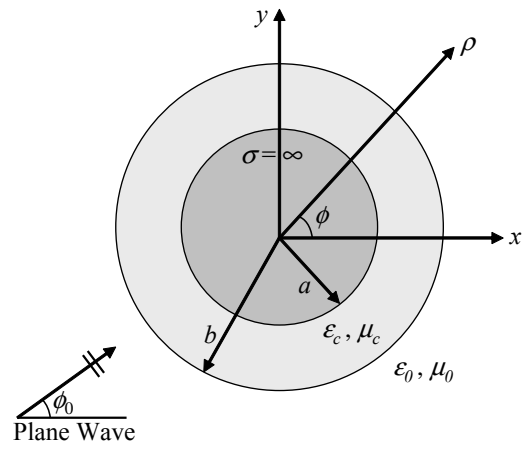

Figure 1: Cross section view of metamaterial coated PEC cylinder.

$$
\begin{gathered}
E_{z}^{t}=E_{0} \sum_{n=-\infty}^{+\infty} j^{-n}\left[a_{n}^{T M} J_{n}\left(k_{c} \rho\right)+b_{n}^{T M} Y_{n}\left(k_{c} \rho\right)\right] e^{j n\left(\phi-\phi_{0}\right)}, \\
E_{z}^{s}=E_{0} \sum_{n=-\infty}^{+\infty} j^{-n} c_{n}^{T M} H_{n}^{(2)}\left(k_{0} \rho\right) e^{j n\left(\phi-\phi_{0}\right)}
\end{gathered}
$$

where $k_{c}=\omega \sqrt{\mu_{c} \varepsilon_{c}}$ is the wave number in the metamaterial coating. The unknown coefficients $a_{n}^{T M}, b_{n}^{T M}$ and $c_{n}^{T M}$ are determined from the simultaneous solution of boundary conditions. Far field expression for the scattered electric field is obtained using the large argument approximation of Hankel functions. Normalized bistatic echo width is then found as

$$
\sigma^{T M} / \lambda_{0}=\lim _{\rho \rightarrow \infty}\left[2 \pi \rho \frac{\left|E_{z}^{s}\right|^{2}}{\left|E_{z}^{i}\right|^{2}}\right] / \lambda_{0}=\frac{2}{\pi}\left|\sum_{n=-\infty}^{+\infty} c_{n}^{T M} e^{j n\left(\phi-\phi_{0}\right)}\right|^{2} .
$$

The procedure for $T E^{z}$ polarization is the same with $T M^{z}$ case, except electric fields are replaced by magnetic fields and corresponding boundary conditions are utilized.

The transparency condition for an electrically small cylindrical scatterer, which is composed of two concentric layers of different isotropic materials, is given in Eq. (10) of [1] for the $T E^{z}$ polarization. When the core cylinder is PEC $(\varepsilon \rightarrow-j \infty)$, this transparency condition becomes $\gamma=\sqrt[2 n]{\frac{\varepsilon_{0}-\varepsilon_{c}}{\varepsilon_{0}+\varepsilon_{c}}}$ for $n \neq 0$, where $\gamma=a / b$ is the ratio of core-shell radii, $n=0,1, \ldots$ is the index of infinite series summation. Since $0<\gamma<1$ should be, transparency interval is found to be $0<\varepsilon_{c}<\varepsilon_{0}$. The aforementioned transparency condition can be rewritten as $\varepsilon_{c}=\frac{1-\gamma^{2 n}}{1+\gamma^{2 n}} \varepsilon_{0}$ to find the coating permittivity for a desired $\gamma$ value, analytically. However, our numerical investigations show that, modifying the original transparency condition heuristically as $\varepsilon_{c}=\frac{1-\gamma^{(2 n-\gamma)}}{1+\gamma^{(2 n-\gamma)}} \varepsilon_{0}$ relates $\varepsilon_{c}$ to $\gamma$ more accurately.

The resonance condition, which increases the RCS drastically for an electrically small cylindrical scatterer, is given in Eq. (8) of [2] for the $T E^{z}$ polarization. Again in the PEC limit, the resonance condition becomes $\gamma=\sqrt[2 n]{\frac{\varepsilon_{0}+\varepsilon_{c}}{\varepsilon_{0}-\varepsilon_{c}}}$ for $n>0$. The valid interval of $\gamma$ (i.e., $0<\gamma<1$ ) restricts the resonance interval to $-\varepsilon_{0}<\varepsilon_{c}<0$. For electrically small cylinders, the resonance condition gives good numerical results to relate $\gamma$ to $\varepsilon_{c}$, so no modification is done. 


\section{Numerical Results and Conclusions}

To verify our numerical routines, we have duplicated the numerical results given in [6]. One of these numerical results is shown in Fig. 2. In addition to the DPS and DNG coatings investigated in [6] (marked by diamonds in Fig. 2), we also included ENG and MNG coatings. As seen in Fig. 2, we have an excellent agreement with the results of [6]. Moreover, there is a perfect continuation in the monostatic echo widths when the coating medium changes to single-negative (SNG) from DPS or DNG, except for the small region where calculation is not possible since $\varepsilon_{c}=0$.

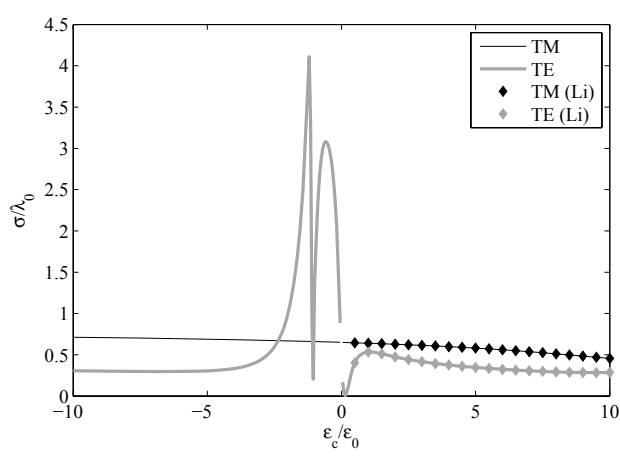

(a) $\mu_{c}=\mu_{0}$

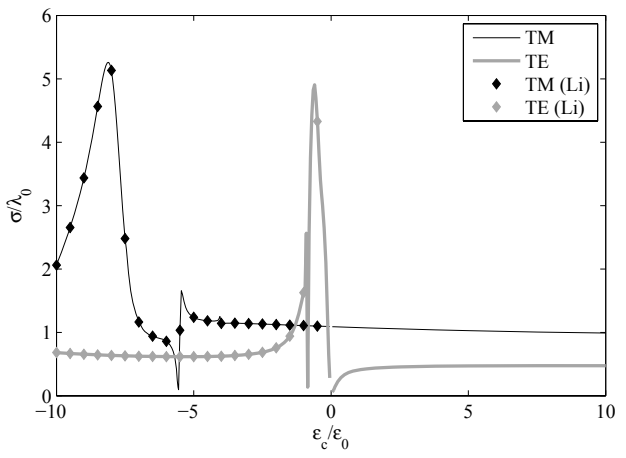

(b) $\mu_{c}=-\mu_{0}$

Figure 2: Normalized monostatic echo width of a metamaterial coated PEC cylinder $(a=50 \mathrm{~mm}, b=70 \mathrm{~mm}, f=1 \mathrm{GHz})$.

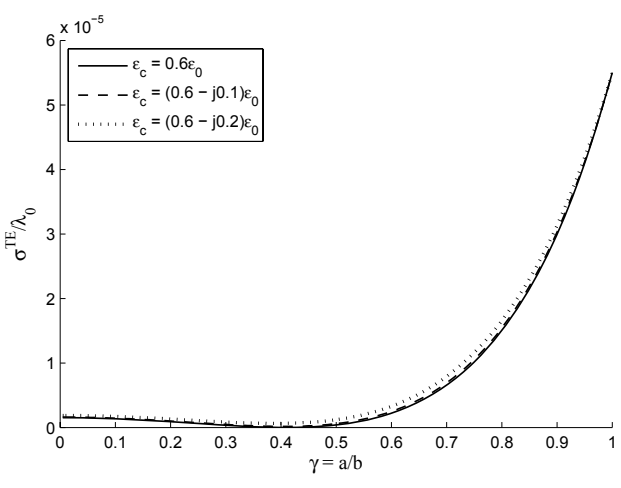

(a) $\varepsilon_{c}=0.6 \varepsilon_{0}, \mu_{c}=\mu_{0}$

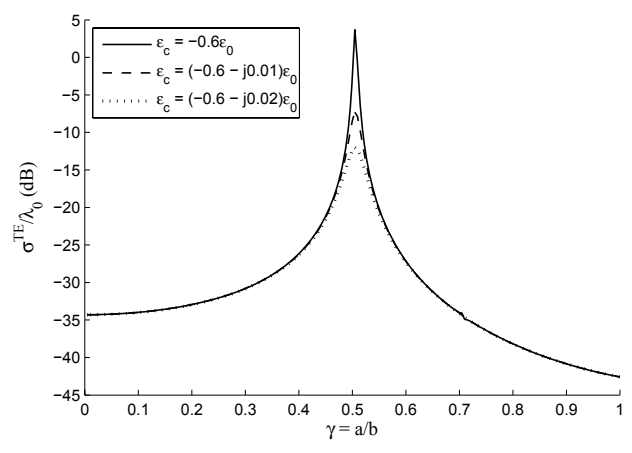

(b) $\varepsilon_{c}=-0.6 \varepsilon_{0}, \mu_{c}=\mu_{0}$

Figure 3: Effects of ohmic losses on normalized monostatic echo width for (a) DPS [transparency] (b) ENG [RCS maximization] cases $\left(b=\lambda_{0} / 100\right)$.

The transparency and resonance (RCS maximization) phenomena are demonstrated in Fig. 3. For both cases, transparency or resonance is desired to be obtained at $\gamma=0.5$. The original transparency relation yields $\varepsilon_{c}=0.6 \varepsilon_{0}$ and the resonance relation yields $\varepsilon_{c}=-0.6 \varepsilon_{0}$ for the metamaterial coating. Since the cylindrical scatter is electrically very small ("quasielectrostatic" problem), we simply select $\mu_{c}=\mu_{0}$ for convenience. Fig. 3(a) shows that transparency is obtained at $\gamma=0.41$ in the lossless case (with our heuristic formula transparency is obtained at $\gamma=0.49$ ). 
The effects of small ohmic losses, as in the Drude or Lorentz medium models, are also shown in Fig. 3. It can be concluded that transparency condition is not very sensitive to ohmic losses. However, the resonance phenomenon is very sensitive. Even there is degradation due to losses, Fig. 3(b) shows an increase of approximately $20 \mathrm{~dB}$ for the most lossy case given.

The bistatic normalized echo widths are also calculated and shown in Fig. 4(a) and Fig. 4(b), respectively for transparency and resonance cases. For transparency, it can be concluded that echo width is minimized in the backscattering direction and increases gradually towards the direction of incidence. In the resonance case, echo width is maximized not only in the backscattering direction but also in the direction of incidence.

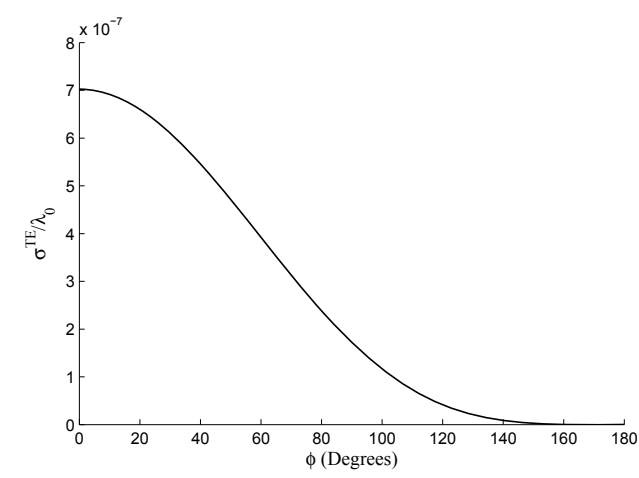

(a) $\varepsilon_{c}=0.6 \varepsilon_{0}, \mu_{c}=\mu_{0}, \gamma=0.41$

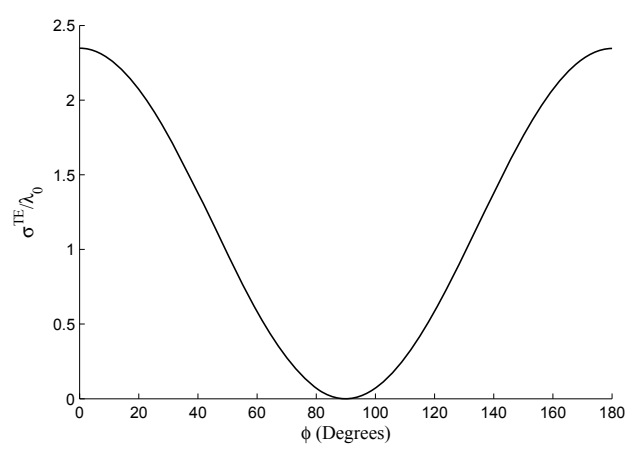

(b) $\varepsilon_{c}=-0.6 \varepsilon_{0}, \mu_{c}=\mu_{0}, \gamma=0.505$

Figure 4: Normalized bistatic echo widths for (a) DPS coated (b) ENG coated PEC cylinder for the $T E^{z}$ polarization case $\left(b=\lambda_{0} / 100, \phi_{0}=0^{\circ}\right)$.

\section{References}

[1] A. Alù and N. Engheta, "Achieving transparency with plasmonic and metamaterial coatings," Phys. Rev. E, vol. 72, pp. 016 623/1-9, July 2005.

[2] — , "Resonances in sub-wavelength cylindrical structures made of pairs of doublenegative and double-negative or epsilon-negative and mu-negative coaxial shells," in Proc. Int. Conf. on Electromagnetics in Advanced Applications, Torino, Italy, Sep. 8-12 2003, pp. 435-438.

[3] — , "Sub-wavelength resonant structures containing double-negative (DNG) or singlenegative (SNG) media: Planar, cylindrical and spherical cavities, waveguides, and open scatterers," in Progress in Electromagnetic Research Symp., Waikiki, HI, Oct. 13-16 2003, p. 12.

[4] — - "Polarizabilities and effective parameters for collections of spherical nano-particles formed by pairs of concentric double-negative (DNG), single-negative (SNG) and/or double-positive (DPS) metamaterial layers," J. Appl. Phys, vol. 97, pp. 094310/1-12, Apr. 2005.

[5] C. A. Balanis, Advanced Engineering Electromagnetics. New York: Wiley, 1989, ch. 11, pp. $595-596$.

[6] C. Li and Z. Shen, "Electromagnetic scattering by a conducting cylinder coated with metamaterials," Progress In Electromagnetics Research, vol. 42, pp. 91-105, 2003. 